\title{
The Role of Computational Epidemiology and Risk Analysis in the Fight Against HIV/AIDS
}

Berhanu Tameru*, David Nganwa, Asseged Bogale, Vinaida Robnett and Tsegaye Habtemariam

Tuskegee University, College of Veterinary Medicine, Nursing and Allied Health, Center for Computational Epidemiology, Bioinformatics and Risk Analysis (CCEBRA), Tuskegee, 36088, USA

\begin{abstract}
Substantial progress in the understanding of HIV and CD4 cell dynamics using computational models undergirded by sound epidemiologic and mathematical principles has been achieved. The early stages of the applications of these models were based on relatively simple mathematical models that considered the body as a one-compartment system. In spite of these models attractiveness due to the experimental and/or mathematical standpoints, the underlying simplification neglected a lot of important factors affecting the population dynamics both on macro (human) and micro (cellular) population levels. This simplification also affected the kinetics linked to the immunology, infection and chemotherapy dynamics throughout the host. Epidemiologic research involves the study of a complex set of host, environmental and causative agent factors as they interact to impact health and diseases in any given population whether biotic or abiotic. This leads in generating large data sets which require the use of powerful computational methods for studying these large and complex models by means of computational epidemiologic methods. Another dimension of a great challenging problem to public health decision makers is that of emerging diseases, as they have to face and deal with a lot of uncertainty at the early stages of disease outbreaks. However, at this juncture, epidemiologic problem-solving and decision-making often proceeds in the face of uncertainties and limited information. One methodology to address these types of shortcomings is the application of risk analysis. Risk analysis is a process for decision making under uncertainty that consists of three fundamental tasks: risk management, risk assessment, and risk communication. Excitingly, the prospective role that computational models and risk analysis may possibly play in the advancement of the theoretical understanding of disease processes and the identification of specific intervention strategies holds the potential to impact and save human lives.
\end{abstract}

Keywords: Computational/Mathematical epidemiology; Risk analysis; Decision making

\section{Introduction}

Computational models and simulations are emerging as vital research tools in epidemiology, biology, and various other fields in advancing the bench (wet) lab and public health policy research agenda. Scientists are recognizing the huge potential of these tools in solving some of today's biggest health problems. Computational epidemiology is a multidisciplinary field utilizing techniques from computer science, mathematics, geographic information science and public health to develop tools and models to aid epidemiologists in their study of the temporal-spatial spread of diseases. Research in computational epidemiology is now considered to be an exponentially expanding arena of scientific exploration. In particular HIV/AIDS, where more than 25 million people have so far died from the disease since 1981, making it amongst the most serious threats to global health that we face today; computational epidemiology has played a major role [1]. Epidemic models of infectious diseases date back to Daniel Bernoulli's mathematical analysis of smallpox in 1760 and have been developed extensively since the early 1900s. Mathematical epidemiologic modeling, with the help of computational tools, has provided new insights on such important issues as drug resistance, rate of spread of infection, epidemic trends, and effects of interventions such as treatment and vaccination.

The term computational epidemiology was first coined by Tsegaye Habtemariam (a founding member of the Center for Computational Epidemiology, Bioinformatics and Risk Analysis (CCEBRA)) [2-4] to better understand the complex biomedical systems in diseases like the HIV/AIDS pandemic. Computational epidemiology enables diseases and risk agents of plants, animals and human infectious diseases to be examined and investigated without jeopardizing lives or creating hazards. This relatively young strand of computational science is being used to understand a range of problems from soybean and wheat rust to HIV/AIDS, swine influenza, foot and mouth disease, rift valley fever and bioterrorism to name a few. In light of this, computational epidemiology has the potential to influence global issues that both directly and indirectly affect human health, representing a milestone in modern science. The CCEBRA is a unique facility that has been involved since the early 1980s in groundbreaking work in this niche of science, that of Computational Science. Epidemiologic research involves the study of a complex set of host, environment and causative agent factors, with the most advanced of these efforts focusing on micro (cellular/molecular) and macro (host) population levels.

Computational epidemiologic models of HIV/AIDS provide important insights in population dynamics through studies at the molecular and cellular levels as well as at the human population level $[1,5,6]$. As of today, bench science has not yet achieved a level of success to produce a viable vaccine or effective medication for preventing or treating HIV/AIDS. The alternative research approach of computational

*Corresponding author: Dr. Berhanu Tameru, Center for Computational Epidemiology, Bioinformatics and Risk Analysis (CCEBRA), 107-Williams-Bowie Hall, College of Veterinary Medicine, Nursing and Allied Health, Tuskegee University, Tuskegee AL 36088, USA, Tel: 334-724-4275; Fax: 334-724-4277; E-mail: tameru@mytu.tuskegee.edu

Received July 17, 2012; Accepted July 19, 2012; Published July 22, 2012

Citation: Tameru B, Nganwa D, Bogale A, Robnett V, Habtemariam T (2012) The Role of Computational Epidemiology and Risk Analysis in the Fight Against HIVIAIDS. J AIDS Clinic Res 3:e107. doi:10.4172/2155-6113.1000e107

Copyright: (C) 2012 Tameru B, et al. This is an open-access article distributed under the terms of the Creative Commons Attribution License, which permits unrestricted use, distribution, and reproduction in any medium, provided the original author and source are credited. 
Citation: Tameru B, Nganwa D, Bogale A, Robnett V, Habtemariam T (2012) The Role of Computational Epidemiology and Risk Analysis in the Fight Against HIVIAIDS. J AIDS Clinic Res 3:e107. doi:10.4172/2155-6113.1000e107

Page 2 of 3

modeling (the so called the third dimension of science), has been a priority for researchers in other disciplines.

\section{Approaches}

Several researchers have developed computational tools and mathematical approaches to study the effects of various mitigation methods on HIV at different population-levels. Models for HIV/AIDS can be conceptual, in vivo or in vitro, systems analysis, mathematical, risk analysis or computational just to name a few [1,5-14].

The approaches developed to understand and predict the spread of infectious diseases and the impact of treatment and control strategies range from compartmental models represented by sets of differential equations [7-9]; to highly complex individual-based models which represent daily activities and interconnections of individuals via transmission networks [10]. Compartmental models can be easily solved, but they cannot model adaptive behaviors of individuals and complex interactions of different groups of populations during disease outbreaks. While individual-based models like Agent Based Modeling can capture the spread of diseases with high-fidelity, modeling large populations often resorts to supercomputers and makes it impractical for quick what-if analyses of interventions or treatments under varying different conditions.

Epidemiologic problem-solving and decision-making often proceeds in the face of uncertainties and limited information. Emerging diseases are one such challenging problem to public health decision makers since they encounter a lot of uncertainty at the early stages of disease outbreaks. Risk analysis is a process for decision making under uncertainty that consists of three fundamental tasks: risk management, risk assessment, and risk communication. Risk analysis can be considered as the process of examining the whole of a risk holistically by assessing the risk and its related relevant uncertainties for the purpose of its efficacious management, facilitated by effective communication about the risk. It is a systematic way of gathering, recording, and evaluating information that can lead to recommendations for a decision or action in response to an identified hazard or opportunity for gain. Risk analysis is not a science; it is not certain; it is not a solution; it is not static. While risk assessment has existed in various forms for many years, the process used by US Environmental Protection Agency (EPA) and others was formalized in the pivotal 1983 National Research Council (NRC) report known as the "Red Book" [15]. The Red Book codified the well-known four steps of risk assessment (hazard identification, exposure assessment, dose-response assessment, and risk characterization) and emphasized the necessity of a conceptual distinction between risk assessment, risk management and risk communication. Over the intervening quarter-century, risk assessment has evolved substantially, driven in part by additional NRC reports, EPA, World Trade Organization, and other agency guidelines, and publications in the peer-reviewed literature. There are two major types of risk assessments Qualitative Risk Assessments and Quantitative Risk Assessments (Probabilistic Risk Assessment (PRA)). PRA is used to estimate risk by computing probability distributions to determine what can go wrong, how likely is it to happen, and what are its consequences. Health (human, animal and plant) PRA provides insights into how the risk propagates from the source to the end point (Scenario tree); how likely is each scenario to happen; what will be the consequence (e.g., the number of people potentially infected or killed); the efficacy and weaknesses of different mitigations to reduce the risk and associated consequences. However, there is a need in expanding the use of PRA in human health as most of the human health risk assessments are environmental and food safety in nature.

\section{Conclusion}

The rationale behind relying upon computational epidemiology and risk analysis modeling is that "Epidemiology" has its tentacles in literally all disciplines, such as in mathematics and statistics, molecular epidemiology, socio epidemiology, computational epidemiology etc. In its simplicity, it focuses upon the study of population dynamics, whatever the population may be (biotic, i.e. living or biological entities, as well as abiotic or non-living population entities)." Key to its breadth of application is epidemiology's extension tentacles into computational and risk analysis models, which can be used to examine and communicate a diversity of epidemiological concerns and scenarios. Excitingly, the role that computational and risk analysis models could play in the advancement of the theoretical understanding of disease processes and the identification of specific intervention strategies and scenarios holds the potential to impact and save human lives. Computational epidemiologic models provide insights in examining a variety of computational experimentations. Complimented with computational tools under the frame work of sound epidemiologic principles, risk analysis can help in facilitation of the current and future problem solving mitigations in tackling the problem of HIV/ AIDS by considering multiple scenarios and sensitivity analysis. Such experimentations can help in evaluating scientific questions related to effective strategies in HIV/AIDS drug therapy interventions and associated risky behaviors.

\section{Acknowledgment}

This work is supported by a Research Centers in Minority Institutions (RCMI) Award, 2G12RR03059-16, from the National Center for Research Resources, and Export Project Award from the National Center for Minority Health and Disparities, National Institutes of Health (NIH).

\section{References}

1. Ho DD, Neumann AU, Perelson AS, Chen W, Leonard JM, et al. (1995). Rapid turnover of plasma virions and CD4 lymphocytes in HIV-1 infection. Nature 373: 123-126.

2. Habtemariam T, Ghartey-Tagoe A, Robnett V, Trammel G (1988) Computationa Epidemiology - New Research Avenues. Proceedings of the 5th International Symposium on Veterinary Epidemiology and Economics, Published in Acta Veterinaria Scandinavia, Supplementum 84

3. Habtemariam T, Ghartey-Tagoe A, Mamo E, Robnett V (1988) Epidemiologic Modelling of Diseases - a Case Example Using Schistosoma and Trypanosoma. Math Comput Modelling 11: 244-249.

4. Habtemariam T, Oryang D, Robnett V, Obasa M (1991) Computational epidemiology : integrating systems analysis and expert systems International Symposia on Veterinary Epidemiology and Economics (ISVEE) proceedings, ISVEE 6: Proceedings of the 6th International Symposium on Veterinary Epidemiology and Economics, Ottawa, Canada, Modelling \& systems analysis session 538-540.

5. Perelson AS, Nelson PW (1999) Mathematical analysis of HIV-1 dynamics in vivo. SIAM Rev 41: 3-44.

6. Wei X, Ghosh SK, Taylor ME, Johnson VA, Emini EA, et al. (1995) Viral dynamics in human immunodeficiency virus type 1 infection. Nature 373: 117122.

7. Anderson RM, May RM (1992) Infectious Diseases of Humans: Dynamics and Control. Oxford University Press, Oxford.

8. Rvachev LA, Longini IM Jr (1985) A mathematical model for the global spread of influenza. Mathematical BioSciences 75: 3-22.

9. Sattenspiel L, Simon CP (1988) The spread and persistence of infectious diseases in structured populations. Mathematical BioSciences 90: 341-366.

10. Eubank S, Guclu H, Kumar VS, Marathe MV, Srinivasan A, et al. (2004) Modelling disease outbreaks in realistic urban social networks. Nature 429 180-184. 
Citation: Tameru B, Nganwa D, Bogale A, Robnett V, Habtemariam T (2012) The Role of Computational Epidemiology and Risk Analysis in the Fight Against HIVIAIDS. J AIDS Clinic Res 3:e107. doi:10.4172/2155-6113.1000e107

Page 3 of 3

11. Habtemariam T, Yu P, Oryang D, Nganwa D, Ayanwale O, et al. (2001) Modelling viral and CD4 cellular population dynamics in HIV: approaches to evaluate intervention strategies. Cell Mol Biol (Noisy-le-grand) 47: 1201-1208.

12. Wai-Yuan T and Hulin W (2008) Deterministic and Stochastic Models of AIDS Epidemics and HIV Infections with Intervention. Hackensack, NJ: World Scientific Publishing Company.

13. Tameru B, Habtemariam T, Nganwa D, Yanwale L, Beyene G, et al. (2008)
Computational Modelling of Intracellular Viral Kinetics and CD4+ Cellular Population Dynamics of HIV/AIDS. Adv Syst Sci Appl 8: 40-45.

14. Gerbi GB, Habtemariam T, Tameru B, Nganwa D, Robnett V (2012)A quantitative risk assessment of multiple factors influencing HIVIAIDS transmission through unprotected sex among HIV-seropositive men. AIDS Care 24: 331-339.

15. National Research Council (1983) Risk Assessment in the Federal Government: Managing the Process. Washington, DC: National Academy Press. 